\title{
Do we need to re-think the obesity issue?
}

\author{
Manfred J. Müller ${ }^{1}$ M. Soares $\mathbb{B}^{2}$
}

Received: 30 October 2018 / Accepted: 30 October 2018 / Published online: 26 February 2019

C) Springer Nature Limited 2019

Obesity is today's no. 1 public health issue. Worldwide obesity has a high prevalence, as well as carries a high risk of several chronic diseases [1-3]. A recent analysis of prospective data obtained in 10 European cohort studies has added to the evidence by examining the relationship of BMI at start and the loss in disease-free years in obese subjects over $\sim 11$-year period [4]. From age 40 to 75 years, this loss was 3.9 and 2.7 years (for obesity class 1 males and females, respectively) and reached up to 8.5 and 7.3 years in obese classes 2 and 3 males and females [4]. The loss of life expectancy was in line with previous data [5, 6]. However, the very striking finding was that the loss in disease-free life years was comparable between subjects from high and low socio-economic status (SES), between smokers and nonsmokers and between those with low and high levels of physical activity [4]. In effect, grades of obesity (as measured by BMI) resulted in the greatest loss of disease-free years compared to the effect of known modifiers considered in this association. Clearly, there are limitations to this analysis as acknowledged by the authors [4]. Further, the effects of diet (and their change) over the years of observation, would have some influence on the outcomes discussed. So do these data provide evidence for the idea that obesity per se, is a determinant of major non-communicable diseases (NCDs)? And, are such data important for developing prevention strategies for obesity, as well as NCDs related to obesity [7]?

Taking a closer look at the impact of lifestyle variables, e.g., at the effects size of physical activity on the number of disease-free years, gives rise to some food for thought. In normal weight and physical active subjects mean age reached prior to disease, was 70 years (men) and 70.9 years

Manfred J. Müller

mmueller@ nutrfoodsc.uni-kiel.de

1 Department of Human Nutrition and Food Science, ChristianAlbrechts-University of Kiel, Kiel, Germany

2 School of Public Health, Curtin University, Perth, WA, Australia (women) compared with 67.6 and 70.1 years in men/women with low physical activity [4]. Thus, it implies the mean effect of regular physical activity is an increase of 2.4 and 0.8 disease-free life years in men and women, respectively. By contrast, in obese classes 2 and 3 subjects the corresponding age data are 62.1 and 63.2 years in physically active men and women compared with 58.8 and 61.7 years in men and women with low physical activity. Thus, in obese subjects the impact of physical activity was 3.3 and 1.5 disease-free years. When compared to the loss of disease-free years lost due to obesity per se, the effect of physical activity could be considered as moderate.

The data suggest that the obesity-related loss in life expectancy is at least in part independent of lifestyle factors, such as physical activity. Thus, there is need of re-thinking our current lifestyle-obesity paradigm, which still performs the basis of conventional strategies followed in prevention and treatment of obesity. As already mentioned in the accompanying editorial to that paper, causality rather than prediction should be a suitable basis of future strategies to prevent and treat obesity [7]. Assuming a causal link between obesity and NCDs suggests that preventing or treating obesity will also be a sound strategy to prevent and treat NCDs. Alternatively, lifestyle (i.e. diet, physical activity, inactivity, smoking, alcohol consumption, smoking, and other factors) may determine both, obesity as well as NCDs. Then, the link between obesity, risk factors, and NCDs become complex. E.g., in the long-term increasing or decreasing physical activity may have different effects on obesity and NCDs. Even more sophisticated one may assume that obesity is associated with the pathophysiology (i.e., it is part of NCDs) rather than being a direct cause of NCDs [7]. Consequently, obesity reflects the joint influence of lifestyle variables on NCDs. If so, future prevention strategies should focus on lifestyle (i.e. health promotion) rather than on obesity itself.

Discussing the present study [4] also refers to the limits of epidemiological research and the need of reform it [8]. Today epidemiological associations between lifestyle factors, overweight and NCDs perform the basis of mortality 
risk assessment, disease prediction algorithms, guidelines of prevention and treatment and subsequently, health policies and health claims. These are based on the idea that associations observed in (mostly) cross-sectional and prospective observational studies can be taken as causal links. However, epidemiological data suggest that nearly all lifestyle variables (including food and nutrient intake data) are correlated with one another, as well as with all outcomes of interest. Then the epidemiological basis of our present understanding becomes more and more complex, and the most abundant determinants (e.g., fats and carbohydrates in the diet) are considered as major risk factors. By contrast the apparent complexity and its reduction to major determinants are considered as questionable because of varying effects of age, genetic background, and environment all together arguing in favor of personalized strategies rather than general recommendations [8]. There is also the increased scrutiny of epidemiological research because of considerable methodological issues, e.g, cumulative bias, confounding, and selective reporting. These limitations cannot be overcome by big data and by the never ending meta-analyses that are presented to every journal. Overall, and to hold the epidemiologist's own view, scientists became part of the problem.

It is obvious that there is need to reconsider the basis of our present understanding of NCDs and to reform the research focus on those issues. Since (i) the epidemiological associations between lifestyle variables (and also other assumed determinants like genes [9]) and obesity are weak, or at best moderate and (ii) treatment and prevention strategies aimed at obesity have rather limited or even no sustained effects [7], there is need to accept that our present view is too narrow to reach solutions. Obviously, we have to keep trying and a solution-oriented approach is wanted. Re-thinking obesity may serve as a model for this process. If obesity becomes part of the disease it should not be considered as a risk factor anymore. As mentioned in our previous editorial 'Now, more than ever, we need to subscribe to tangible solutions for our global problems' [10], and 'Re-iterating old messages is not helpful' [10]. Given this context we feel that the present study [4] may be taken as a renewed starting point to go beyond our present understanding of how to tackle this global health issue.

\section{Compliance with ethical standards}

Conflict of interest The authors declare that they have no conflict of interest.

\section{References}

1. NCD Risk Factor Collaboration (NCD-RisC). Worldwide trends in body-mass index, underweight, overweight, and obesity from 1975 to 2016: a pooled analysis of 2416 population-based measurement studies in 128.9 million children, adolescents, and adults. Lancet. 2017;390:2627-42.

2. Kivimäki M, Kuosma E, Ferrie JE, Luukkonen R, Nyberg ST, Alfredsson L, et al. Overweight, obesity, and risk of cardiometabolic multimorbidity: pooled analysis of individual-level data for 120813 adults from 16 cohort studies from the USA and Europe. Lancet Public Health. 2017;2:e277-e285.

3. Müller MJ, Soares MJ. How many more numbers do we need? Eur J Clin Nutr. 2017;71:1363-4.

4. Nyberg ST, Batty GD, Pentti J, Luukkonen R, Nyberg ST, Alfredsson L, et al. Obesity and loss of disease-free years owing to major non-communicable diseases: a multicohort study. Lancet Public Health. 2018;3:e490-497.

5. Prospective Studies Collaboration. Body mass index and causespecific mortality in 900000 adults: collaborative analyses of 57 prospective studies. Lancet. 2009;373:1083-96.

6. Flegal KM, Kit BK, Orpana H, Graubard BI. Association of allcause mortality with overweight and obesity using standard body mass index categories: a systematic review and meta-analysis. JAMA. 2013;309:71-82.

7. Chiolero A. Why causality, and not prediction, should guide obesity prevention policy. Lancet Public Health. 2018;3:e461e462.

8. Ioannidis JPA. The challenge of reforming nutritional epidemiologic research. JAMA.https://doi.org/10.1001/jama.2018.11025 (published online data 23 Aug 2018)

9. Müller MJ, Geisler C, Blundell J, Dulloo A, Schutz Y, Krawczak $\mathrm{M}$, et al. The case of GWAS of obesity: does body weight control play the rules? Int J Obesity. 2018. https://doi.org/10.1038/ s41366-018-0081-6

10. Soares M, Müller MJ. Thirty years of EJCN: a time for reflection. Eur J Clin Nutr. 2018;72:1195-1197. 